\title{
Double heterodyne interferometer using a multimode laser diode
}

\author{
S. Han, E. Dalhoff, E. Fischer, S. Kreuz and H. J. Tiziani \\ Institute for Applied Optics, University Stuttgart \\ Pfaffenwaldring 9, 70569 Stuttgart, Germany
}

\begin{abstract}
A double heterodyne interferometer using a multimode laser diode with a synthetic wavelength of $\sim 1 \mathrm{~mm}$ has been established and the stability of the synthetic wavelength has been investigated.

\section{INTRODUCTION}

Several kinds of lasers have been used in multiwavelength interferometry, e. g. a multiwavelength $\mathrm{HeNe}^{1}$, a monomode laser diode with acustooptical modulator (AOM), or several independent emitting lasers ${ }^{2,3}$. If the application in question is to perform a measurement on the synthetic wavelength at relatively large distances rather than making measurements, which are performed on one wavelength, unambiguous around the point of equal optical paths, the main task is to create a synthetic wavelength of sufficient stability. In principle the stability of the effective wavelength which is generated by two modes of a multimode laser is better than that of several monomode lasers'; provided the stability of a single modes of the multimode laser equals the stability of the modes of the two free running lasers. To overcome the stability problem of two free running laser expensive electronic and/or optical coupling schemes are needed, which makes the usage especially of cheap multimode laser diodes (MMLD) attractive. In this paper we report a two-wavelength double heterodyne interferometer (DHI) using a MMLD.
\end{abstract}

\section{PRINCIPLES OF OPERATION}

A double heterodyne interferometer consists of two independent heterodyne interferometers working at different wavelengths $\lambda_{1}$ and $\lambda_{2}$ and different heterodyne frequencies $f_{1}$ and $f_{2}$. Two wavelengths can be obtained from the multimode laser diode. After amplitude demodulation and band-pass filtering of the superimposed interference signals we get the low frequency signal $i(t)^{\prime}$,

$$
i(t) \propto \cos \left(2 \pi\left(f_{1}-f_{2}\right) t+\left(\Phi_{1}-\Phi_{2}\right)\right)
$$

The interference phase difference $\Phi_{1}-\Phi_{2}$ that depends on the wanted synthetic wavelength corresponds now to the target distance $z$.

$$
\Phi=\left|\Phi_{1}-\Phi_{2}\right|=4 \pi z\left|\frac{1}{\lambda_{1}}-\frac{1}{\lambda_{2}}\right|=\frac{4 \pi z}{\Lambda}
$$

Eq. (2) indicates the relationship among phase difference, the target distance and the synthetic wavelength. The distance sensitivity thus is reduced and the range of unambiguity for interferometric measurement is extended. Particularly, the fluctuation $\Delta \Lambda_{\text {mmld }}$ of the synthetic wavelength caused by fluctuation of the driving current and the temperature is smaller than the fluctuation $\Delta \Lambda_{2 l d}$ of the synthetic wavelength generated by two monomode laser diodes', because the two wavelengths of the multimode laser diode are amplified by the same cavity.

$$
\frac{\Delta \Lambda_{\text {mmld }}}{\Delta \Lambda_{2 l d}}=\frac{\lambda}{\sqrt{2} \Lambda} .
$$

One of the main advantages of using a multimode laser diode in the double heterodyne interferometer is indicated by eq. (3). 


\section{EXPERIMENT}

Fig. 1 is a sketch of a two-wavelength double heterodyne interferometer. The light of the MMLD (Sony model SLD202U-3) is split by a sinusoidal grating (SG), the positive and negative first diffraction-orders of it being focussed by two lenses. In the

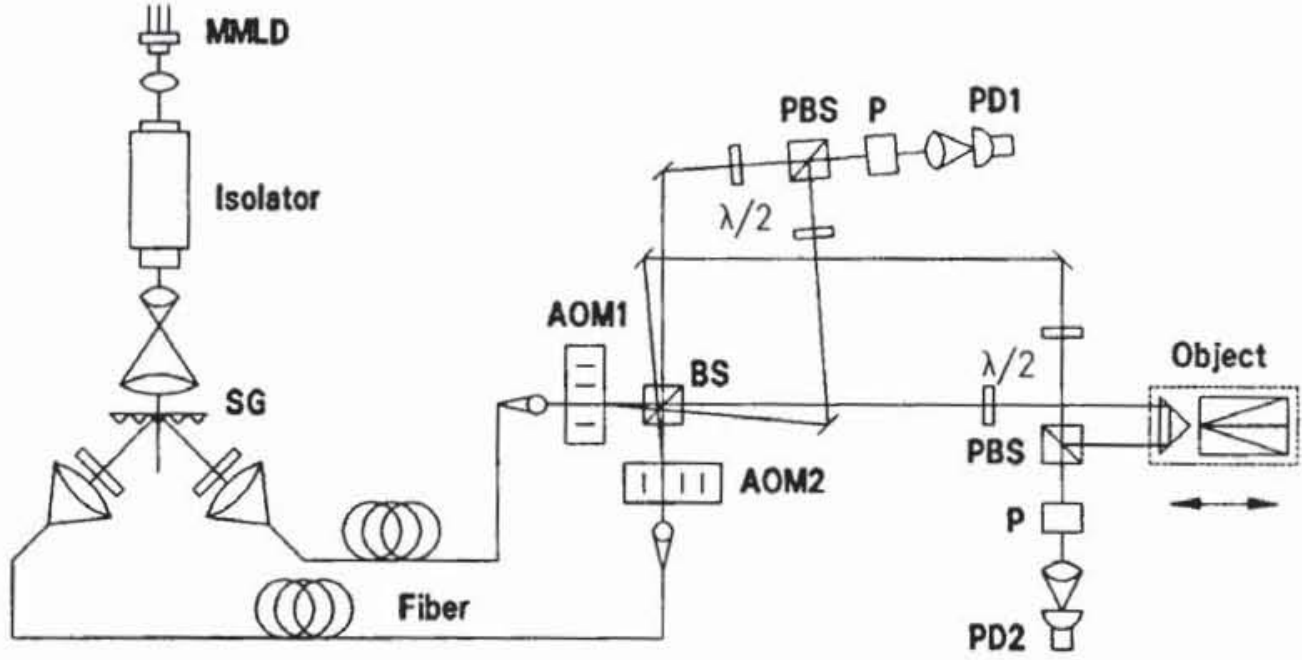

Fig. 1 Experimental setup

focal planes the light of one longitudinal mode of the laser diode can be selected and coupled into a monomode fiber. The two beams with different wavelengths $\lambda_{1}$ and $\lambda_{2}$ are shifted by the correspondent heterodyne frequencies $f_{1}$ and $f_{2}$ using two $\mathrm{AOMs}$ and are combined in a beam splitter (BS). Also the two zero orders are superimposed and serve as object beams. The object and the reference light is then superimposed twice, i.e. feeding two photodetectors (PD), which give the signals of a measuring and a control interferometer.

\section{RESULTS}

Fig. 2 shows the spectrum of two selected modes of the MMLD with the wavelengths $813.1 \mathrm{~nm}$ and $813.8 \mathrm{~nm}$, constituting a synthetic wavelength of $1.02 \mathrm{~mm}$ (observed from an optical spectrum analyzer). Of course other synthetic wavelength can be chosen, the largest possible being approximately $2 \mathrm{~mm}$.

The stability of the phase difference over 100 seconds is shown in Fig. 3. The root-mean-square (RMS) of the phase difference measurement is $0.06^{\circ}$ (corresponding to a distance resolution of $0.085 \mu \mathrm{m}$ ).

Fig. 4 shows a distance measurement on a corner cube, which has been shifted every ten seconds. The movement was measured from behind by an HP interferometer model $5526 \mathrm{~A}$.

Fig. 5 shows the phase stability of the interferometer signal dependent on the balance of the optical paths. By means of a Fabry-Perot interferometer the width of a single mode of the multimode laser diode was measured to be $15 \mathrm{GHz}$, the coherent length $\left(=\lambda^{2} / \Delta \lambda\right)$ thus is $20 \mathrm{~mm}$. In this experiment the optimal value of $<0.1^{\circ}$ at zero position was not obtained due to alignement problems. 


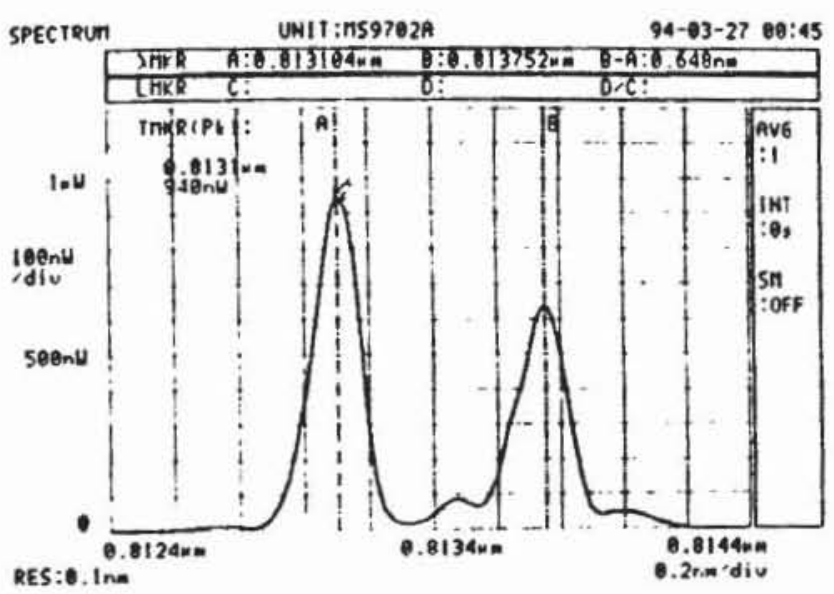

Fig.2 Two separated wavelenghts from the MMLD

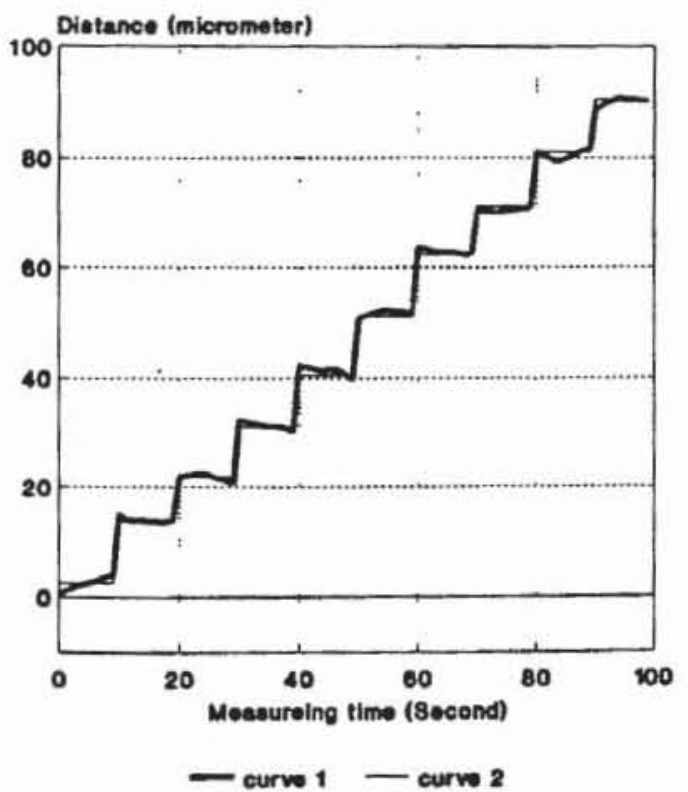

Fig. 4 Distance measurement, (1) measured by the HP interferometer; (2) measured by the DHI

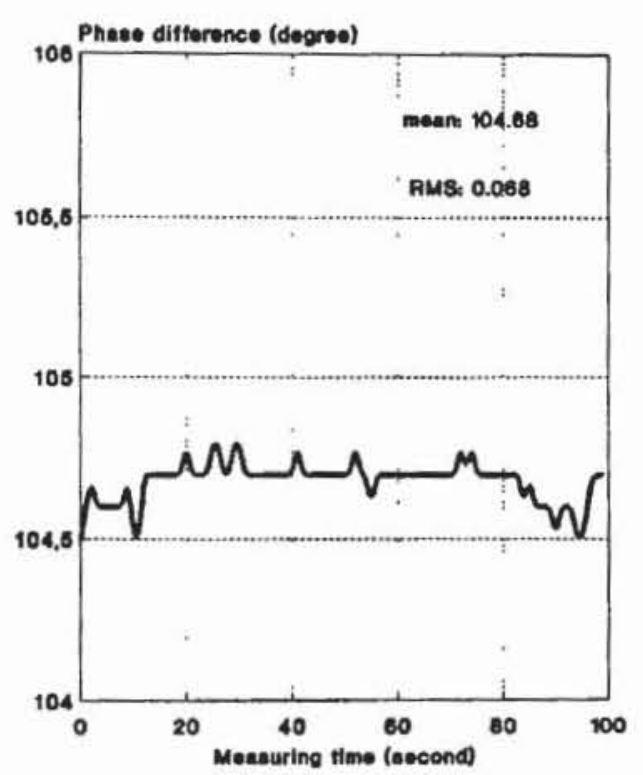

Fig. 3 Stability of the phase difference

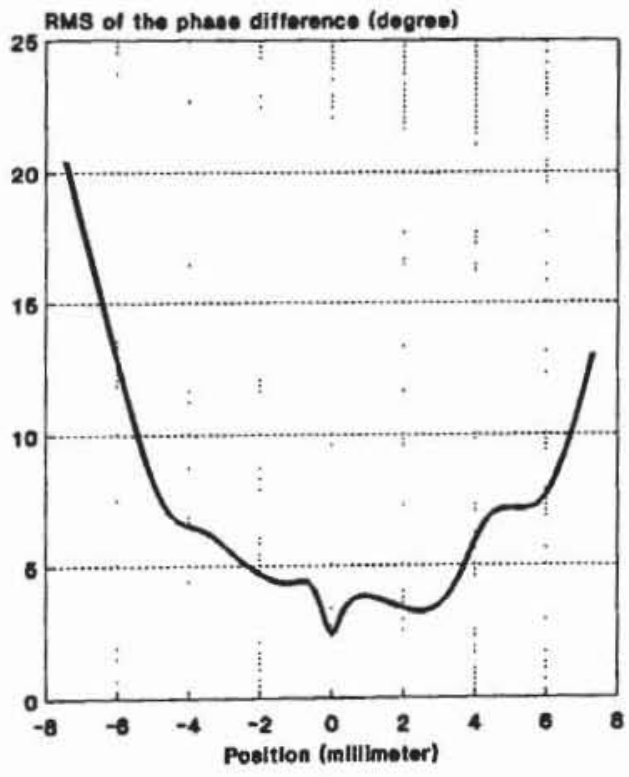

Fig. 5 Balance measurement 


\section{CONCLUSION}

The stability of the synthetic wavelength established by two modes of a multimode laser diode has been investigated. Using a synthetic wavelength of $\sim 1 \mathrm{~mm}$ a distance resolution of $0.085 \mu \mathrm{m}$ has been obtained. With a path imbalance of $2 \cdot z=12$ $\mathrm{mm}$ corresponding to more than half the coherence length a resolution of $\sim 10^{\circ}$ has been obtained.

\section{ACKNOWLEDGEMENTS}

S. Han, a Deutscher Akademischer Austauschdienst (DAAD) fellow, wishes to thank the DAAD foundation for financial support.

\section{Z.REFERENCES}

1. E. Fischer et al.," Heterodynverfahren fuer hochgenaue Vermessung im Nahbereich," Zeitschrift fuer Vermessung, 1, 46 (1992)

2. J.C. Wyant, "Testing Aspherics Using Two-Wavelength Holography," Appl. Opt. 10, 2113 (1971).

3. C. Polhemus, "Two-Wavelength Interferometry," Appl. Opt. 12, 2071 (1973)

4. S. Han et al., "Effective Wavelength Stability Using a Multimode Laser Diode in DH,," (not published).

5. Peter de Groot, "Three-Color Laser-Diode Interferometer," Appl. Opt. 30, 3612 (1991) 